\title{
The Geometry on a Step 3 Grushin Model
}

Dedicated to Professor B.-Wolfgang. Schulze on his sixtieth birthday

Ovidiu Calin, Eastern Michigan University, Department of Mathematics, Ypsilanti, MI, 48197, USA, ocalin@emunix.emich.edu

Der-Chen Chang, Georgetown University, Department of Mathematics, Washington DC 2005\%-0001, USA, chang@math.georgetown.edu *

April 15, 2004

\begin{abstract}
In this article we study the geometry associated with the sub-elliptic operator $\frac{1}{2}\left(X_{1}^{2}+X_{2}^{2}\right)$, where $X_{1}=\partial_{x}$ and $X_{2}=\frac{x^{2}}{2} \partial_{y}$ are vector fields on $\mathbf{R}^{2}$. We show that any point can be connected with the origin by at least one geodesic and we provide an approximate formula for the number of the geodesics between the origin and the points situated outside of the $y$-axis. We show there are infinitely many geodesics between the origin and the points on the $y$-axis.
\end{abstract}

Key words: Grushin operator, subRiemannian geometry, geodesics, HamiltonJacobi theory, elliptic functions, Euler's theta functions, Jacobi's epsilon function. MS Classification (2000): Principal: 53C17; Secondary: 34K10, 35H20

\section{Introduction}

Consider the following vector fields on $\mathbf{R}^{2}$

$$
X_{1}=\frac{\partial}{\partial x}, \quad X_{2}=\frac{x^{2}}{2} \frac{\partial}{\partial y}
$$

*Partially supported by a William Fulbright Research grant and a competitive research grant at Georgetown University 
and construct the Grushin operator (see $[\mathbf{1 3}]$ )

$$
\Delta_{G}=\frac{1}{2}\left(X_{1}^{2}+X_{2}^{2}\right)=\frac{1}{2}\left(\frac{\partial^{2}}{\partial x^{2}}+\frac{x^{4}}{4} \frac{\partial^{2}}{\partial y^{2}}\right) .
$$

Note that $X_{1}$ and $X_{2}$ are linearly independent everywhere except on the $y$-axis, where $X_{2}$ vanishes. Consequently, the operator $\Delta_{G}$ is elliptic except on the $y$-axis. On the other hand, $\left[X_{1},\left[X_{1}, X_{2}\right]\right]=\frac{\partial}{\partial y}$ which is step 3 on the line $x=0$. Therefore, Chow's theorem [11] holds and every two points on the $x, y$-plane can be connected by a piecewise differentiable horizontal curve. A horizontal curve is a curve whose tangents are linear combinations of $X_{1}$ and $X_{2}$. More precisely, given any two points $P$ and $Q$ in $\mathbf{R}^{2}$, there exists a curve $\mathcal{C}$ connecting these two points such that

$$
\dot{\mathcal{C}}=a_{1} X_{1}+a_{2} X_{2}
$$

Then

$$
\ell(\mathcal{C})=\int_{0}^{\tau} \sqrt{a_{1}^{2}(s)+a_{2}^{2}(s)} d s
$$

is the length of $\mathcal{C}$. By minimizing the lengths of horizontal curves between $P$ and $Q$, we obtain the distance between these two points. Furthermore, we may apply Hörmander's theorem $[\mathbf{1 4}]$ to conclude that $\Delta_{G}$ is hypoelliptic. There is a significant difference between the elliptic and non-elliptic cases. As we can see from Riemannian geometry (which corresponds to elliptic theory), every point is connected to all nearby points by a unique geodesic. This is no longer true in the sub-elliptic case. A very careful study of the subRiemannian geometry on Heisenberg groups [6] shows that every point in the center of the group is connected to the origin by an infinite number of geodesics of different lengths. A similar situation happens in some other cases, see e.g., [8], [9], and [10]. This strange phenomenon was first pointed out by Gaveau [12] and Strichartz [16], and it brings up the question of what "local" means in subRiemannian geometry. Control theorists studying subRiemannian exampls noticed that the Riemannian concepts of cut locus and conjugate locus behave badly in a subRiemannian context.

In this article, we shall use Hamilton-Jacobi theory of bicharacteristics to study some geometric properties induced by the operator $\Delta_{G}($ see $[\mathbf{1}],[\mathbf{2}]$, $[\mathbf{3}],[\mathbf{5}]$ and $[\mathbf{7}])$. We obtain the following results:

Theorem 1.1 Given a point $P(0, \mathbf{y})$, there are infinity many geodesics between the origin and $P$. Their lengths are given by

$$
\ell_{m}^{3}=\frac{3|\mathbf{y}| m^{2}}{4 \pi} \cdot \Gamma\left(\frac{1}{4}\right)^{4}, m=1,2, \ldots
$$


Theorem 1.2 Let $\mathbf{y} / \mathbf{x}^{3}>0$. There are finitely many geodesics between the origin and the point $(\mathbf{x}, \mathbf{y})$ with $\mathbf{x} \neq 0$.

If $\mathbf{y} / \mathbf{x}^{3}$ is small enough there is only one geodesic.

If $\mathbf{y} / \mathbf{x}^{3}$ is large enough the number $N$ of geodesics is approximated by

$$
N \approx 2\left[\frac{3}{\sqrt{2} K} \frac{\mathbf{y}}{\mathbf{x}^{3}}-\frac{1}{4}\right] .
$$

These theorems generalize the results of $[\mathbf{6}]$ and $[\mathbf{9}]$ to the step 3 Grushin operator $\Delta_{G}$. Moreover, our case is quite different from the Heisenberg group. For a step 2 case, one needs only elementary functions. However, it requires the use of elliptic functions in our case which makes the calculation much more complicated.

Part of this article is based on a lecture presented by the second author during the Spring School of EU Research and Training Network on "Geometric Analysis" which was held on March 1-6, 2004 at Institute of Mathematics, Universität of Potsdam, Germany. The second author would like to thank the organizing committee, especially Professor B.-Wolfgang. Schulze for his warm hospitality during his visit to Germany.

\section{$2 \quad$ Elliptic functions}

The Hamiltonian of the operator $\Delta_{G}$ can be considered as follows

$$
H(x, y, \theta, \xi)=\frac{1}{2}\left(\frac{1}{4} x^{4} \theta^{2}+\xi^{2}\right)
$$

where $\xi$ and $\theta$ are the dual variables of $x$ and $y$. The geodesics between the origin $O$ and the point $P(x, y)$ are the projections on the $(x, y)$-plane of the solutions of the Hamilton's system of equations

$$
\left\{\begin{array}{l}
\dot{x}=H_{\xi}=\xi \\
\dot{y}=H_{\theta}=\frac{1}{4} x^{4} \theta \\
\dot{\xi}=-H_{x}=-\frac{1}{2} x^{3} \theta^{2} \\
\dot{\theta}=-H_{y}=0,
\end{array}\right.
$$

with the boundary conditions

$$
x(0)=\mathbf{x}_{0}, y(0)=\mathbf{y}_{0}, x(1)=\mathbf{x}_{1}, y(1)=\mathbf{y}_{1} .
$$

In order to solve the system (2.3) with boundary values (2.4), one needs to use elliptic functions. Before we go further, let us recall some basic 
properties of elliptic functions which will be used in this paper. For detailed discussions, readers may consult the book by Lawden [15].

The integral

$$
z=\int_{0}^{w} \frac{d t}{\sqrt{\left(1-t^{2}\right)\left(1-k^{2} t^{2}\right)}}, \quad|k|<1
$$

is called an elliptic integral of the first kind. The integral exists if $w$ is real and $|w|<1$. Using the substitution $t=\sin \theta$ and $w=\sin \phi$

$$
z=\int_{0}^{\phi} \frac{d \theta}{\sqrt{1-k^{2} \sin ^{2} \theta}} .
$$

If $k=0$, then $z=\sin ^{-1} w$ or $w=\sin z$. By analogy, the above integral is denoted by $\mathrm{sn}^{-1}(w ; k)$, where $k \neq 0$. $k$ is called the modulus. Thus

$$
z=\mathrm{sn}^{-1} w=\int_{0}^{w} \frac{d t}{\sqrt{\left(1-t^{2}\right)\left(1-k^{2} t^{2}\right)}} .
$$

The function $w=\operatorname{sn} z$ is called a Jacobian elliptic function.

By analogy with the trigonometric functions, it is convenient to define other elliptic functions

$$
\operatorname{cn} z=\sqrt{1-\operatorname{sn}^{2} z}, \quad \operatorname{dn} z=\sqrt{1-k^{2} \operatorname{sn}^{2} z} .
$$

A few properties of this functions are

$$
\begin{gathered}
\operatorname{sn}(0)=0, \quad \operatorname{cn}(0)=1, \quad \operatorname{dn}(0)=1, \\
\operatorname{sn}(-z)=\operatorname{sn}(z), \quad \operatorname{cn}(-z)=\operatorname{cn}(z), \\
\frac{d}{d z} \operatorname{sn} z=\operatorname{cn} z \operatorname{dn} z, \frac{d}{d z} \operatorname{cn} z=-\operatorname{sn} z \operatorname{dn} z, \frac{d}{d z} \operatorname{dn} z=-k^{2} \operatorname{sn} z \operatorname{cn} z \\
-1 \leq \operatorname{cn} z \leq 1, \quad-1 \leq \operatorname{sn} z \leq 1, \quad 0 \leq \operatorname{dn} z \leq 1
\end{gathered}
$$

Let

$$
K=K(k)=\int_{0}^{1} \frac{d t}{\sqrt{\left(1-t^{2}\right)\left(1-k^{2} t^{2}\right)}}=\int_{0}^{\pi / 2} \frac{d \theta}{\sqrt{1-k^{2} \sin ^{2} \theta}}
$$

be the complete elliptic integral. Then, as real functions, the elliptic functions sn and $\mathrm{cn}$ are periodic functions of principal period $4 K$. 


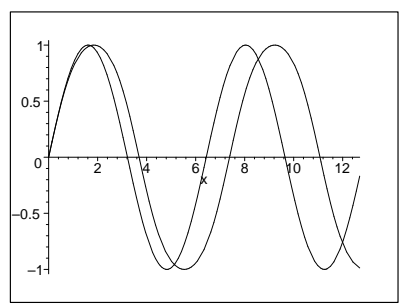

$\operatorname{sn}(z, k)$

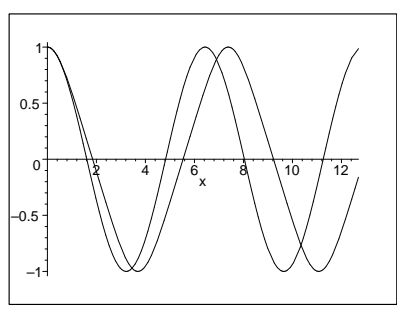

$\operatorname{cn}(z, k)$

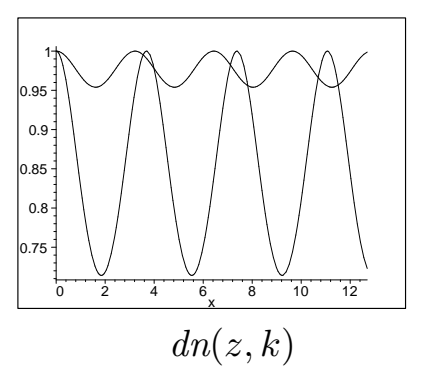

$d n(z, k)$

Figure 1: The graphs of functions $\operatorname{sn}(z, k), \operatorname{cn}(z, k)$ and $d n(z, k)$ for $k=0.3$ and 0.7

\section{Solving the Hamiltonian system}

In this section we find explicit formulas for the geodesics between the origin and the point $(x, y)$. Similar formulas are obtained by Agrachev, Bonnard, Chyba and Kupka in the Martinet case on $\mathbf{R}^{3}$, see [1]. We make use of elliptic functions which can be found for instance in Lawden [15]. From (2.3), we know that $\theta=H_{y}=0$. Hence the momentum $\theta$ is a constant which can be considered as a Lagrange multiplier. We have the following theorem.

Theorem 3.1 For any two points $P\left(\mathbf{x}_{0}, \mathbf{y}_{0}\right)$ and $Q\left(\mathbf{x}_{1}, \mathbf{y}_{0}\right)$ on the same horizontal line $y=\mathbf{y}_{0}$, there is only one geodesic

$$
x(s)=s\left(\mathbf{x}_{1}-\mathbf{x}_{0}\right)+\mathbf{x}_{0}, \quad y(s)=\mathbf{y}_{0}, \quad s \in[0,1]
$$

connecting them. The length of the geodesic is $\left|\mathbf{x}_{1}-\mathbf{x}_{0}\right|$.

Proof: From the Hamiltonian system we have $\dot{y}(s)=0, \dot{x}(s)=$ constant. Hence the geodesic should have the form (3.6). Moreover, from the the second equation of (2.3), one has $\dot{y}=\frac{1}{2} \theta x^{4}$. Then

(i) $y$ is increasing if $\theta>0$,

(ii) $y$ is decreasing if $\theta<0$,

(iii) $y=$ constant $=0$, if $\theta=0$.

The cases $(i)$ and $(i i)$ are not possible because $y(0)=y(1)=\mathbf{y}_{0}$. Hence the $y$-component is fixed and the momentum $\theta$ must be zero. Then $\ddot{x}=$ $-\frac{1}{2} x^{3} \theta^{2}=0$ and $x(s)=s\left(\mathbf{x}_{1}-\mathbf{x}_{0}\right)+\mathbf{x}_{0}$. Therefore, the length of the geodesic is $|x(1)-x(0)|=\left|\mathbf{x}_{1}-\mathbf{x}_{0}\right|$.

As a consequence, given a point $P(\mathbf{x}, 0), \mathbf{x} \neq 0$, there is a unique geodesic joining the origin and the point $P$. This geodesic is a straight segment line 
with length equal to $|\mathbf{x}|$. Now let us turn to the case $\theta \neq 0$. The Hamilton's system is invariant by the symmetries

$$
(x, y ; \theta) \rightarrow(-x, y ; \theta), \quad(x, y ; \theta) \rightarrow(x,-y ;-\theta) .
$$

These symmetries will sent geodesics into geodesics. For this reason we shall study only the case $x>0, y>0$ and $\theta>0$, unless otherwise stated. Since the operator is translation invariant along the $y$-axis, we may assume the boundary conditions along the $y$ direction are $y(0)=\mathbf{y}_{0}=0$ and $y(1)=$ $\mathbf{y}_{1}-\mathbf{y}_{0}=\mathbf{y}$. Moreover, in this paper we just study geodesics start from the origin, i.e., $\mathbf{x}_{0}=0$. Hence (2.4) can be rewritten as

$$
x(0)=y(0)=0, \quad x(1)=\mathbf{x}, \quad y(1)=\mathbf{y}_{1}-\mathbf{y}_{0}=\mathbf{y} .
$$

\subsection{The $x$-component.}

- Conservation of energy. The first equation of (2.3) yields $\dot{x}=H_{\xi}=\xi$. Hence $\ddot{x}=\dot{\xi}=-H_{x}=-\frac{1}{2} \theta^{2} x^{3}$. Then $x(s)$ satisfies

$$
\ddot{x}=-\frac{1}{2} x^{3} \theta^{2},
$$

with boundary conditions $x(0)=0, x(1)=\mathbf{x}$. We have $\theta=$ constant, because $\dot{\theta}=-H_{y}=0$. Let $V=\frac{x^{4}}{8} \theta^{2}$ be the potential. Then (3.7) can be written as a Newton equation $\ddot{x}=-V^{\prime}(x)$. The law of conservation of energy is

$$
\frac{1}{2} \dot{x}^{2}+\frac{x^{4}}{8} \theta^{2}=E \text { (the constant of energy). }
$$

- The arc length parametrization. Consider the metric in which the vector fields $X_{1}$ and $X_{2}$ are orthonormal. Let $\mathcal{C}=(x(s), y(s))$ be a curve. The velocity is

$$
\dot{\mathcal{C}}=\dot{x} \partial_{x}+\dot{y} \partial_{y}=\dot{x} X_{1}+\frac{2 \dot{y}}{x^{2}} X_{2}
$$

The square of the length in the above metric is

$$
|\dot{\mathcal{C}}|^{2}=\dot{x}^{2}+\frac{4 \dot{y}^{2}}{x^{4}}=\dot{x}^{2}+\frac{x^{4} \theta^{2}}{4}=2 E,
$$


where we have used the second equation of (2.3) and (3.8). Let $s$ be the arc length parameter. Then $E=\frac{1}{2}$ and

$$
\dot{x}(s)^{2}+\frac{x^{4}(s)}{4} \theta^{2}=1 .
$$

$x(s)$ is defined by the integral

$$
\int_{0}^{x(s)} \frac{d u}{\sqrt{1-\frac{u^{4}}{4} \theta^{2}}}=s .
$$

Making the substitution $v=\sqrt{\frac{\theta}{2}} u, \quad d u=\sqrt{\frac{2}{\theta}} d v$, and

$$
\int_{0}^{\sqrt{\frac{\theta}{2}} x(s)} \frac{d v}{\sqrt{\left(1-v^{2}\right)\left(1+v^{2}\right)}}=\sqrt{\frac{\theta}{2}} s .
$$

This can be written in terms of Jacobi elliptic functions as follows (see Lawden [15] p.53)

$$
\frac{1}{\sqrt{2}} \operatorname{sd}^{-1}\left(\sqrt{2} \cdot \frac{\sqrt{\theta}}{\sqrt{2}} x(s), \frac{1}{\sqrt{2}}\right)=\sqrt{\frac{\theta}{2}} s,
$$

where $\operatorname{sd}(z)=\frac{\operatorname{sn}(z)}{\operatorname{dn}(z)}$. Solving for $x(s)$, yields

$$
x(s)=\frac{1}{\sqrt{\theta}} \operatorname{sd}\left(\sqrt{\theta} s, \frac{1}{\sqrt{2}}\right) .
$$

Following [15], p.28, one has $\operatorname{cn}(u+K)=-k^{\prime} \operatorname{sd} u$, where $k^{2}+{k^{\prime}}^{2}=1$. In our case $k=k^{\prime}=\frac{1}{\sqrt{2}}$, and hence

$$
x(s)=-\sqrt{\frac{2}{\theta}} \operatorname{cn}\left(\sqrt{\theta} s+K, \frac{1}{\sqrt{2}}\right),
$$

where $K=K\left(\frac{1}{\sqrt{2}}\right)$ is the complete elliptic integral defined by (2.5). From Lawden [15], p.103,

$$
K=K\left(\frac{1}{\sqrt{2}}\right)=\int_{0}^{1} \frac{\sqrt{2} d t}{\sqrt{\left(1-t^{2}\right)\left(1+t^{2}\right)}}=\frac{1}{4} \pi^{-\frac{1}{2}}\left[\Gamma\left(\frac{1}{4}\right)\right]^{2} \approx 1.854 .
$$




\section{$3.2 \quad$ The $y$-component}

Integrating the Hamiltonian equation $\dot{y}(s)=H_{\theta}=\frac{1}{4} x^{4}(s) \theta$ yields

$$
\begin{aligned}
y(s) & =\frac{\theta}{4} \int_{0}^{s} x^{4}(u) d u=\frac{1}{\theta} \int_{0}^{s} \operatorname{cn}^{4}(\sqrt{\theta} u+K) d u \\
& =\frac{1}{\theta} \int_{K}^{\sqrt{\theta} s+K} \operatorname{cn}^{4} w \frac{1}{\sqrt{\theta}} d w=\frac{1}{\theta \frac{3}{2}} \int_{K}^{\sqrt{\theta} s+K} \operatorname{cn}^{4} w d w
\end{aligned}
$$

where we used the substitution $w=\sqrt{\theta} u+K$. Following Lawden [15], p.87 $\int \mathrm{cn}^{4} w d w=\frac{1}{3 k^{4}}\left[\left(2-3 k^{2}\right) k^{2} w+2\left(2 k^{2}-1\right) E(w)+k^{2} \operatorname{sn} w \cdot \operatorname{cn} w \cdot \operatorname{dn} w\right]$.

Here $E(w, k)$ is the Jacobi's epsilon function defined by

$$
E(w, k)=\frac{d}{d w}\left(\log \left(\theta_{4}\right)\right)+\frac{E w}{K}
$$

where

$$
E=\left[1-\frac{\theta_{4}^{\prime \prime}(0)}{\theta_{3}^{4}(0) \theta_{4}(0)}\right] K
$$

with $\theta_{3}$ and $\theta_{4}$ are Euler's theta functions and $K$ is defined by (3.10). When $k=\frac{1}{\sqrt{2}}=k^{\prime}, 2 k^{2}-1=0$, the above formula yields

$$
\begin{aligned}
\int \operatorname{cn}^{4}\left(w, \frac{1}{\sqrt{2}}\right) d w & =\frac{4}{3}\left[\frac{w}{4}+\frac{1}{2} \operatorname{sn} w \cdot \operatorname{cn} w \cdot \operatorname{dn} w\right] \\
& =\frac{1}{3}[w+2 \operatorname{sn} w \cdot \operatorname{cn} w \cdot \operatorname{dn} w]
\end{aligned}
$$

It follows that

$$
\begin{aligned}
y(s) & =\left.\frac{1}{3 \theta^{\frac{3}{2}}}(w+2 \operatorname{sn} w \cdot \operatorname{cn} w \cdot \operatorname{dn} w)\right|_{K} ^{\sqrt{\theta} s+K} \\
& =\frac{1}{3 \theta^{\frac{3}{2}}}(\sqrt{\theta} s+2 \operatorname{sn}(\sqrt{\theta} s+K) \cdot \operatorname{cn}(\sqrt{\theta} s+K) \cdot \operatorname{dn}(\sqrt{\theta} s+K))
\end{aligned}
$$

and hence

$$
y(s)=\frac{2}{3 \theta^{\frac{3}{2}}}\left(\frac{1}{2} \sqrt{\theta} s+\operatorname{sn} u \cdot \operatorname{cn} u \cdot \operatorname{dn} u\right),
$$


where $u=\sqrt{\theta} s+K$. With this notation, relation (3.9) becomes

$$
x(s)=-\sqrt{\frac{2}{\theta}} \operatorname{cn} u .
$$

Formulas (3.13) and (3.12) depend on two parameters $s$ and $\theta$, the first being the arc length and the second a momentum. Each solution $(s, \theta)$ of the system

$$
\left\{\begin{array}{l}
x(s, \theta)=\mathbf{x} \\
y(s, \theta)=\mathbf{y}
\end{array}\right.
$$

defines a geodesic between $(0,0)$ and $(\mathbf{x}, \mathbf{y})$. In the following sections we describe the number of solutions $(s, \theta)$ of the system (3.14). There are two cases: $\mathbf{x}=0$ and $\mathbf{x} \neq 0$.

\section{The geodesics in the case $x=0$}

In this section we shall obtain infinitely many geodesics of distinct lengths. It is known that the period of the function $\mathrm{cn}$ is $4 K$. We also know that $\mathrm{cn}(4 m K)=1$ and $\mathrm{cn}(2 m K+K)=0$ for $m=0,1,2, \ldots$ If $\mathbf{x}=0$, formula (3.13) yields $K+\sqrt{\theta} s=K+2 m K$, or

$$
\sqrt{\theta} s=2 m K \text {. }
$$

As cn $u=0$, relation (3.12) yields

$$
y(s)=\frac{2}{3 \theta^{\frac{3}{2}}} \cdot \frac{1}{2} \sqrt{\theta} s=\frac{2}{3 \theta^{\frac{3}{2}}} \cdot \frac{2 m K}{2}=\frac{2 m K}{3 \theta^{\frac{3}{2}}} .
$$

Hence

$$
\mathbf{y}=\frac{2 m K}{3 \theta^{\frac{3}{2}}},
$$

from where we obtain the parameter $\theta$ as the following

$$
\sqrt{\theta}=\left(\frac{2 m K}{3 \mathbf{y}}\right)^{\frac{1}{3}},
$$

where $K=K\left(\frac{1}{\sqrt{2}}\right)$. The parameter $s$, which is the arc length, follows from equation (4.15)

$$
\ell=\frac{2 m K}{\sqrt{\theta}}=(3 \mathbf{y})^{\frac{1}{3}} \cdot(2 m K)^{\frac{2}{3}} .
$$

Using the expression for $K=K(1 / \sqrt{2})$ in terms of Gamma functions given by (3.10) we get the following result. 
Theorem 4.1 Given $\mathbf{y} \neq 0$, there are infinitely many geodesics between the origin and $(0, \mathbf{y})$. Their lengths are given by

$$
\ell_{m}^{3}=\Gamma\left(\frac{1}{4}\right)^{4} \cdot \frac{3|\mathbf{y}| m^{2}}{4 \pi}, \quad m=1,2, \ldots
$$

\section{$5 \quad$ The geodesics in the case $\mathrm{x} \neq 0$}

In this section we investigate the number of geodesics between the origin and any point $(\mathbf{x}, \mathbf{y})$ with $\mathbf{y} \neq 0$. The study will be done in two steps. First we make the computations under the assumption $2 K>u>K$. We shall reduce the system (3.14) to an equation of one variable which can be analyzed by standard techniques. Let $\sigma=\sqrt{\theta}$. Then (3.12) and (3.13) can be written as

$$
\begin{gathered}
\operatorname{cn} u=-\frac{\mathbf{x} \sigma}{\sqrt{2}}, \\
\frac{3 \mathbf{y}}{2} \sigma^{3}=\frac{1}{2} \sigma s+\operatorname{sn} u \cdot \operatorname{cn} u \cdot \operatorname{dn} u \\
\Leftrightarrow 3 \mathbf{y} \sigma^{3}=\underbrace{(\sigma s+K)}_{=u}-K+2 \operatorname{sn} u \cdot \operatorname{cn} u \cdot \operatorname{dn} u .
\end{gathered}
$$

Case $u \in[K, 2 K)$

As $u=\sigma s+K$, then $u \geq K$. Assuming $2 K>u \geq K$, then equation (5.16) can be inverted using a formula from Lawden $[\mathbf{1 5}]$ p.52,

$$
u=\int_{c n u}^{1} \frac{d z}{\left(1-z^{2}\right)^{\frac{1}{2}} \cdot\left(k^{\prime 2}+k^{2} z^{2}\right)^{\frac{1}{2}}}=\int_{\frac{-\mathbf{x} \sigma}{\sqrt{2}}}^{1} \frac{d z}{\left(1-z^{2}\right)^{\frac{1}{2}}\left(\frac{1}{2}+\frac{1}{2} z^{2}\right)^{\frac{1}{2}}} .
$$

As

$$
K=K\left(\frac{1}{\sqrt{2}}\right)=\int_{0}^{1} \frac{d z}{\left(1-z^{2}\right)^{\frac{1}{2}}\left(\frac{1}{2}+\frac{1}{2} z^{2}\right)^{\frac{1}{2}}},
$$

using the substitution $\frac{z}{\sqrt{2}}=v, d z=\sqrt{2} d v$, we get

$$
\begin{aligned}
u-K & =\int_{0}^{\frac{\mathbf{x} \sigma}{\sqrt{2}}} \frac{d z}{\sqrt{\left(1-z^{2}\right)\left(\frac{1}{2}+\frac{1}{2} z^{2}\right)}} \\
& =\int_{0}^{\frac{\mathbf{x} \sigma}{2}} \frac{d v}{\sqrt{\left(\frac{1}{2}-v^{2}\right)\left(\frac{1}{2}+v^{2}\right)}}
\end{aligned}
$$


Using the formula for $u$ we get

$$
u-K=s \sqrt{\theta}=s \sigma=\int_{0}^{\frac{\mathbf{x} \sigma}{2}} \frac{d z}{\sqrt{\left(\frac{1}{2}-z^{2}\right) \cdot\left(\frac{1}{2}+z^{2}\right)}} .
$$

Make the substitution $\gamma=\frac{\mathbf{x} \sigma}{2}$. Then $0 \leq \gamma \leq \frac{1}{\sqrt{2}}$. Equation (5.16) becomes

$$
\operatorname{cn} u=-\frac{\mathbf{x} \sigma}{\sqrt{2}}=-\gamma \sqrt{2}
$$

As $2 K>u>K$, then $\operatorname{sn} u>0$ and

$$
\begin{aligned}
\operatorname{sn} u & =\sqrt{1-\operatorname{cn}^{2} u}=\sqrt{1-2 \gamma^{2}}, \\
\operatorname{dn} u & =\sqrt{k^{\prime 2}+k^{2} \operatorname{cn}^{2} u}=\sqrt{\frac{1}{2}+\gamma^{2}}=\frac{\sqrt{1+2 \gamma^{2}}}{\sqrt{2}} .
\end{aligned}
$$

Then

$$
2 \operatorname{sn} u \operatorname{cn} u \operatorname{dn} u=-2 \sqrt{2} \gamma \sqrt{1-2 \gamma^{2}} \cdot \frac{\sqrt{1+2 \gamma^{2}}}{\sqrt{2}}=-2 \gamma \sqrt{1-4 \gamma^{4}} .
$$

Substituting (5.19) and (5.21) in formula (5.22) yields

$$
3 \mathbf{y} \sigma^{3}=\int_{0}^{\gamma} \frac{d z}{\sqrt{\left(\frac{1}{2}-z^{2}\right)\left(\frac{1}{2}+z^{2}\right)}}-2 \gamma \sqrt{1-4 \gamma^{4}} .
$$

Let $g(z)=\frac{1 / 2}{\sqrt{\left(\frac{1}{2}-z^{2}\right)\left(\frac{1}{2}+z^{2}\right)}}=\frac{1}{\sqrt{1-4 z^{4}}}$. We shall write the equation (5.22) in function of $\gamma$ only. As $2 \gamma=x \sigma$, then $\sigma^{3}=\frac{8 \gamma^{3}}{\mathbf{x}^{3}}$ and (5.22) becomes

$$
\begin{aligned}
& \frac{3 \mathbf{y} \cdot 8 \gamma^{3}}{\mathbf{x}^{3}}=2 \int_{0}^{\gamma} g(z) d z-2 \gamma \sqrt{1-4 \gamma^{4}} \\
& \Leftrightarrow \frac{12 \mathbf{y}}{\mathbf{x}^{3}} \gamma^{2}=\frac{1}{\gamma} \int_{0}^{\gamma} g(z) d z-\sqrt{1-4 \gamma^{4}}
\end{aligned}
$$

which is an equation of the variable $\gamma$, with $\gamma \in[0,1 / \sqrt{2}]$. To each solution $\gamma$ of the equation (5.24) corresponds an unique $\sigma$ and hence an unique parameter $\theta$. From (5.19) we obtain an unique parameter $s$. Hence to each 
solution $\gamma$ corresponds an unique pair $(s, \theta)$ solution for the system (3.14) i.e., a geodesic between the origin and $(\mathbf{x}, \mathbf{y})$.

In the following we discuss the existence of the solutions of equation (5.24). Denote by

$$
f(\gamma)=\frac{1}{\gamma} \int_{0}^{\gamma} g(z) d z-\sqrt{1-4 \gamma^{4}}
$$

The function $f$ is increasing and $f(0)=g(0)-1=0$. Indeed, differentiating

$$
\begin{aligned}
f^{\prime}(\gamma) & =\frac{\int_{0}^{\gamma}(g(\gamma)-g(z)) d z}{\gamma^{2}}+\frac{8 \gamma^{3}}{\sqrt{1-4 \gamma^{4}}} \\
& =\frac{1}{\gamma^{2}} \cdot \int_{0}^{\gamma} z g^{\prime}(z) d z+\frac{8 \gamma^{3}}{\sqrt{1-4 \gamma^{4}}} \\
& =\frac{8}{\gamma^{2}} \int_{0}^{\gamma} \frac{z^{4}}{\left(1-4 z^{4}\right)^{\frac{3}{2}}} d z+\frac{8 \gamma^{3}}{\sqrt{1-4 \gamma^{4}}} \\
& \geq 0 .
\end{aligned}
$$

As

$$
\lim _{\gamma \rightarrow 0} \frac{\int_{0}^{\gamma} \frac{z^{4}}{\left(1-4 z^{4}\right)^{\frac{3}{2}}} d z}{\gamma^{2}}=\lim _{\gamma \rightarrow 0} \frac{\frac{\gamma^{4}}{\left(1-4 \gamma^{4}\right)^{\frac{3}{2}}}}{2 \gamma}=0
$$

then $f^{\prime}(0)=0$. Next, we shall find $f\left(\frac{1}{\sqrt{2}}\right)$.

$$
f\left(\frac{1}{\sqrt{2}}\right)=\sqrt{2} \int_{0}^{\frac{1}{2}} \frac{d z}{\sqrt{1-4 z^{4}}}=\int_{0}^{1} \frac{d v}{\sqrt{1-v^{4}}} .
$$

From Lawden [15], p.85

$$
\int_{\mathbf{x}}^{1} \frac{d v}{\sqrt{1-v^{4}}}=\frac{1}{\sqrt{2}} \mathrm{cn}^{-1}\left(\mathbf{x}, \frac{1}{\sqrt{2}}\right)
$$

and hence

$$
f\left(\frac{1}{\sqrt{2}}\right)=\int_{0}^{1} \frac{d v}{\sqrt{1-v^{4}}}=\frac{1}{\sqrt{2}} \mathrm{cn}^{-1}\left(0, \frac{1}{\sqrt{2}}\right)=\frac{1}{\sqrt{2}} K\left(\frac{1}{\sqrt{2}}\right) .
$$

Then

$$
f\left(\frac{1}{\sqrt{2}}\right)=\frac{K}{\sqrt{2}}
$$


where

$$
K=K\left(\frac{1}{\sqrt{2}}\right) \approx 1.85
$$

and hence $f\left(\frac{1}{\sqrt{2}}\right)>1$. In the following we shall compute the second derivative of $f$ at zero. The first derivative is

$$
f^{\prime}(\gamma)=\frac{8}{\gamma^{2}} \int_{0}^{\gamma} \frac{z^{4}}{\left(1-4 z^{4}\right)^{\frac{3}{2}}} d z+\frac{8 \gamma^{3}}{\sqrt{1-4 \gamma^{4}}} .
$$

Differentiate each term of $f^{\prime}(\gamma)$ and take $\gamma=0$.

$$
\begin{gathered}
\left(\frac{\gamma^{3}}{\sqrt{1-4 \gamma^{4}}}\right)^{\prime}=\frac{3 \gamma^{2} \sqrt{1-4 \gamma^{4}}-\gamma^{3} \cdot \frac{-8 \gamma^{3}}{\sqrt{1-4 \gamma^{4}}}}{1-4 \gamma^{4}} \\
=\frac{3 \gamma^{2}\left(1-4 \gamma^{4}\right)+8 \gamma^{6}}{\left(1-4 \gamma^{4}\right)^{\frac{3}{2}}}=\frac{3 \gamma^{2}-12 \gamma^{6}+8 \gamma^{6}}{\left(1-4 \gamma^{4}\right)^{\frac{3}{2}}} \\
=\frac{3 \gamma^{2}-4 \gamma^{6}}{\left(1-4 \gamma^{4}\right)^{\frac{3}{2}}}=\frac{\gamma^{2}\left(3-4 \gamma^{4}\right)}{\left(1-4 \gamma^{4}\right)^{\frac{3}{2}}}=0, \text { when } \gamma=0 . \\
\left(\frac{1}{\gamma^{2}} \int_{0}^{\left.\gamma \frac{z^{4}}{\left(1-4 z^{4}\right)^{\frac{3}{2}}} d z\right)^{\prime}}=\frac{1}{\gamma^{4}}\left[\gamma^{2} \frac{\gamma^{4}}{\left(1-4 \gamma^{4}\right)^{\frac{3}{2}}}-2 \gamma \int_{0}^{\gamma} \frac{z^{4}}{\left(1-4 z^{4}\right)^{\frac{3}{2}}} d z\right]\right. \\
=\frac{\gamma^{2}}{\left(1-4 \gamma^{4}\right)^{\frac{3}{2}}}-2 \frac{1}{\gamma^{3}} \int_{0}^{\gamma} \frac{z^{4}}{\left(1-4 z^{4}\right)^{\frac{3}{2}}} d z . \\
\lim _{\gamma \rightarrow 0} \frac{\int_{0}^{\gamma} \frac{z^{4} d z}{\left(1-4 z^{4}\right)^{\frac{3}{2}}}}{\gamma^{3}}=\lim _{\gamma \rightarrow 0} \frac{\left(1-4 \gamma^{4}\right)^{\frac{3}{2}}}{3 \gamma^{2}}=\lim _{\gamma \rightarrow 0} \frac{1}{3} \frac{\gamma^{2}}{\left(1-4 \gamma^{4}\right)^{\frac{3}{2}}}=0 .
\end{gathered}
$$

Hence $f^{\prime \prime}(0)=0$.

Set $h(\gamma)=\frac{12 \mathbf{y}}{\mathbf{x}^{3}} \gamma^{2}$. The equation (5.24) becomes $h(\gamma)=f(\gamma)$. We shall choose $\mathbf{x}>0, \mathbf{y}>0$. The other cases follow from this case using the Lagrangian symmetries. We have

$$
h^{\prime \prime}(0)=\frac{24 \mathbf{y}}{\mathbf{x}^{3}}>0 .
$$

If $h\left(\frac{1}{\sqrt{2}}\right)<f\left(\frac{1}{\sqrt{2}}\right)$, i.e., $\frac{6 \mathbf{y}}{\mathbf{x}^{3}}<\frac{\Gamma\left(\frac{1}{4}\right)^{2}}{4 \sqrt{2 \pi}} \approx 1.31$, then there is a solution $\gamma \in\left(0, \frac{1}{\sqrt{2}}\right)$ for the equation $h(\gamma)=f(\gamma)$. See Figure 1 . 
If $\frac{6 \mathbf{y}}{\mathbf{x}^{3}}>\frac{\Gamma\left(\frac{1}{4}\right)^{2}}{4 \sqrt{2 \pi}}$, there are no solutions $\gamma$ in $\left(0, \frac{1}{\sqrt{2}}\right)$, see Figure 1 . We note that

$$
f^{\prime}\left(\frac{1}{\sqrt{2}}\right)=16 \underbrace{\int_{0}^{\frac{1}{\sqrt{2}}} \frac{z^{4}}{\sqrt{\left(1-2 z^{2}\right)^{3}\left(1+2 z^{2}\right)^{3}}} d z}_{=+\infty}+\underbrace{\frac{4 / \sqrt{2}}{\sqrt{1-1}}}_{=+\infty}=+\infty .
$$

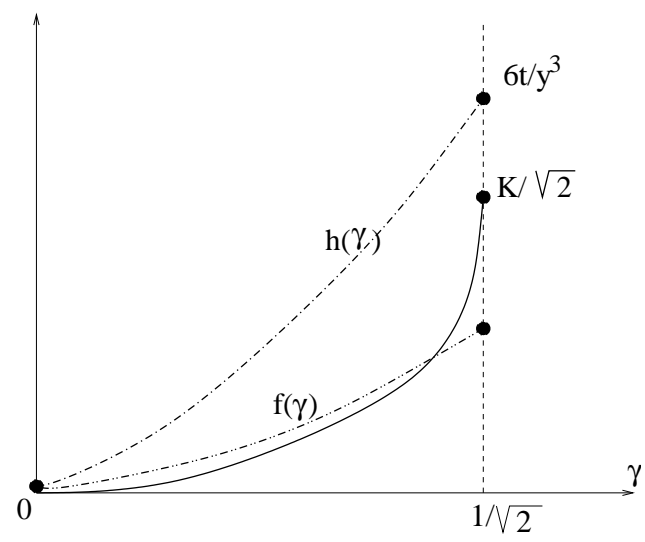

Figure 1: The graphs of $f(\gamma)$ and $h(\gamma)$.

\section{Considering the solutions $\pm u+4 m K$}

Starting with the solution $u \in[K, 2 K)$ for $\operatorname{cn} u=-\frac{\mathbf{x} \sigma}{\sqrt{2}}$, we have arrived at the equation in $\gamma$

$$
\frac{12 \mathbf{y}}{\mathbf{x}^{3}} \gamma^{2}=f(\gamma),
$$

where $f(\gamma)$ is increasing on the interval $(0,1 / \sqrt{2})$.

We consider now all the solutions of (5.16) which are of the form $\pm u+4 m K$, with $u \in[K, 2 K)$, and we shall derive similar equations for $\gamma$.

- Considering the solutions $u+4 m K$, relation (5.18) becomes

$$
\begin{aligned}
3 \mathbf{y} \sigma^{3} & =(u+4 m K)-K+2 \operatorname{sn}(u+4 m K) \operatorname{cn}(u+4 m K) \operatorname{dn}(u+4 m K) \\
\Leftrightarrow 3 \mathbf{y} \sigma^{3} & =u-K+2 \operatorname{sn} u \operatorname{cn} u \operatorname{dn} u+4 m K, \quad m=0,1,2, \ldots
\end{aligned}
$$


Using (5.19) yields

$$
\begin{aligned}
3 \mathbf{y} \sigma^{3} & =\int_{0}^{\frac{\overbrace{\mathbf{x} \sigma}^{2}}{=\gamma}} \frac{d v}{\sqrt{\left(\frac{1}{2}-v^{2}\right)\left(\frac{1}{2}+v^{2}\right)}}+2 \operatorname{sn} u \operatorname{cn} u \operatorname{dn} u+4 m K \\
\Leftrightarrow 3 \mathbf{y} \sigma^{3} & =2 \int_{0}^{\gamma} \frac{d z}{\sqrt{1-4 z^{4}}}-2 \gamma \sqrt{1-4 \gamma^{4}}+4 m K \\
\Leftrightarrow \frac{12 \mathbf{y}}{\mathbf{x}^{3}} \gamma^{2} & =\underbrace{\frac{1}{\gamma} \int_{0}^{\gamma} \frac{d z}{\sqrt{1-4 z^{4}}}-\sqrt{1-4 \gamma^{4}}}_{=f(\gamma)}+\frac{2 m K}{\gamma} .
\end{aligned}
$$

Denote by

$$
f_{m}(\gamma)=f(\gamma)+\frac{2 m K}{\gamma}, \quad m=0,1,2, \ldots
$$

then the above equation becomes

$$
\frac{12 \mathbf{y}}{\mathbf{x}^{3}} \gamma^{2}=f_{m}(\gamma), \quad m=0,1,2, \ldots
$$

Now we shall study the function $f_{m}(\gamma)$ and sketch its graph. One has

$$
\begin{aligned}
f_{m}(0) & =f(0)+\lim _{\gamma \rightarrow 0} \frac{2 m K}{\gamma}=+\infty . \\
f_{m}^{\prime} & =\underbrace{f^{\prime}(0)}_{=0}+\lim _{\gamma \rightarrow 0} \frac{-2 m K}{\gamma^{2}}=-\infty, \\
f_{m}\left(\frac{1}{\sqrt{2}}\right) & =f\left(\frac{1}{\sqrt{2}}\right)+2 \sqrt{2} m K \\
& =\frac{\sqrt{2}}{2} K+2 \sqrt{2} m K=\left(\frac{1}{2}+2 m\right) \sqrt{2} K, \\
f_{m}^{\prime}\left(\frac{1}{\sqrt{2}}\right) & =\underbrace{f^{\prime}\left(\frac{1}{\sqrt{2}}\right)}_{+\infty}+\lim _{\gamma \rightarrow 1 / \sqrt{2}} \frac{-2 m K}{\gamma^{2}}=+\infty .
\end{aligned}
$$


The graph of the function $f_{m}$ is given in Figure 2.

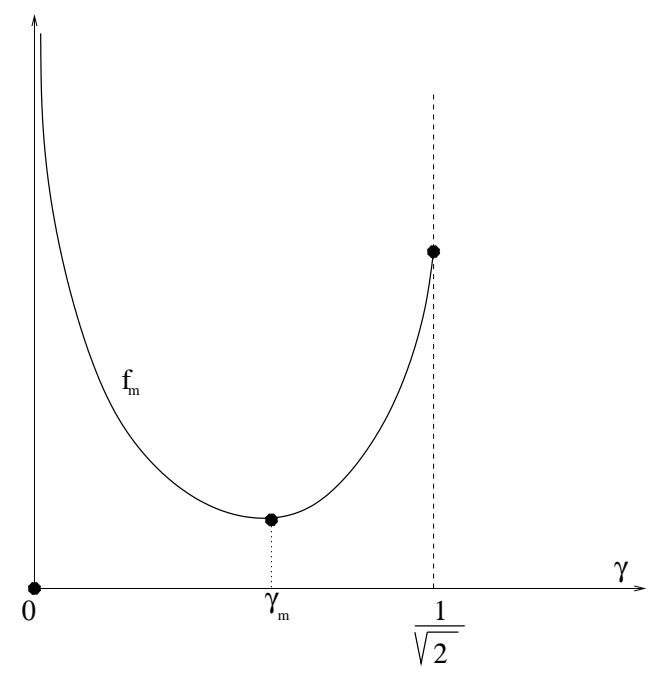

Figure 2: The graph of $f_{m}(\gamma)$.

We still need to show that there is a unique critical point $\gamma_{m}$ (which is a minimum). Indeed, considering the equation $f_{m}^{\prime}(\gamma)=0$, one has

$$
\begin{aligned}
& f^{\prime}(\gamma)=\frac{2 m K}{\gamma^{2}} \\
& \Leftrightarrow \frac{8}{\gamma^{2}} \int_{0}^{\gamma} \frac{z^{4}}{\left(1-4 z^{4}\right)^{3 / 2}} d z+\frac{8 \gamma^{3}}{\sqrt{1-4 \gamma^{4}}}=\frac{2 m K}{\gamma^{2}} \mid \cdot \frac{\gamma^{2}}{8} \\
& \Leftrightarrow \underbrace{\int_{0}^{\gamma} \frac{z^{4}}{\left(1-4 z^{4}\right)^{3 / 2}} d z+\frac{\gamma^{4}}{\sqrt{1-4 \gamma^{4}}}}_{\chi(\gamma) \nearrow}=\frac{m K}{4} .
\end{aligned}
$$

Hence the critical points are solutions of the equation

$$
\chi(\gamma)=\frac{m K}{4} .
$$

The function $\chi(\gamma)$ is strictly increasing and unbounded, with

$$
\chi(0)=0, \quad \chi(1 / \sqrt{2})=+\infty .
$$


Its graph is given in Figure 3.

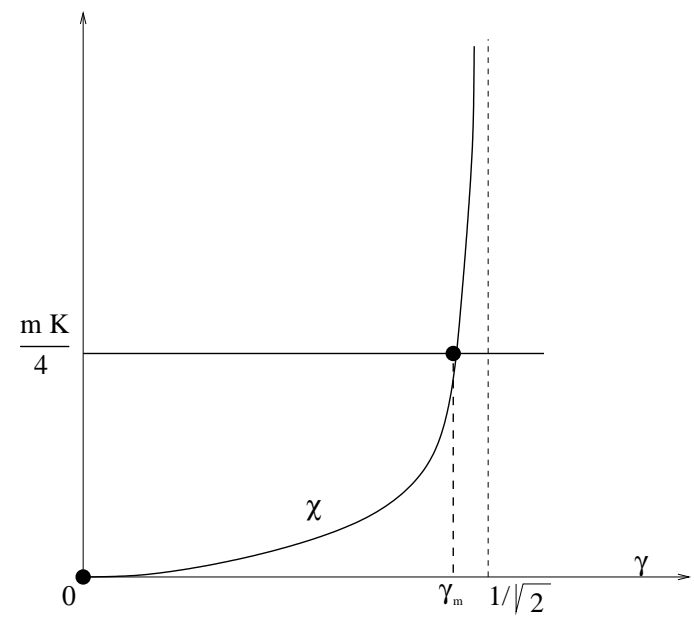

Figure 3: The graph of $\chi(\gamma)$.

Hence there is an unique critical point $\gamma_{m} \in(0,1 / \sqrt{2}), f_{m}^{\prime}\left(\gamma_{m}\right)=0$.

From Figure 3 we note that

$$
\gamma_{m}<\gamma_{m+1}, \quad \lim _{m \rightarrow \infty} \gamma_{m}=\frac{1}{\sqrt{2}} .
$$

The function $f_{m}$ has a minimum value equal to $f_{m}\left(\gamma_{m}\right)$, which tends to infinity

$$
f_{m}\left(\gamma_{m}\right)=\underbrace{f\left(\gamma_{m}\right)}_{\rightarrow f(1 / \sqrt{2})=\frac{1}{\sqrt{2}} K}+\underbrace{2 m K}_{\rightarrow \infty} \underbrace{\frac{1}{\gamma_{m}}}_{\rightarrow \sqrt{2}} \rightarrow+\infty \quad \text { as } m \rightarrow \infty .
$$

- Considering the solutions $-u+4 m K, m=1,2, \ldots$ relation (5.18) becomes

$$
\begin{aligned}
3 \mathbf{y} \sigma^{3} & =(-u+4 m K)-K+2 \operatorname{sn}(-u+4 m K) \operatorname{cn}(-u+4 m K) \operatorname{dn}(-u+4 m K) \\
\Leftrightarrow 3 y \sigma^{3} & =(K-u)+4 m K-2 K-2 \operatorname{sn} u \operatorname{cn} u \operatorname{dn} u \\
\Leftrightarrow 3 \mathbf{y} \sigma^{3} & =-((u-K)+2 \operatorname{sn} u \operatorname{cn} u \operatorname{dn} u)+2(2 m-1) K \\
\Leftrightarrow-\frac{12 \mathbf{y}}{\mathbf{x}^{3}} \gamma^{2} & =\underbrace{f(\gamma)-\frac{2 m-1}{\gamma} K}_{=\tilde{f}_{m}(\gamma)},
\end{aligned}
$$

which can be written as

$$
\frac{12 \mathbf{y}}{\mathbf{x}^{3}} \gamma^{2}=-\tilde{f}_{m}(\gamma), \quad m=1,2,3 \ldots
$$


We shall study the function $\tilde{f}_{m}$ and sketch its graph.

$$
\begin{aligned}
& \tilde{f}_{m}(0)=f(0)-\lim _{\gamma \rightarrow 0^{+}} \frac{(2 m-1) K}{\gamma}=-\infty, \\
& \tilde{f}_{m}^{\prime}(0)=f^{\prime}(0)+\lim _{\gamma \rightarrow 0^{+}} \frac{(2 m-1) K}{\gamma^{2}}=+\infty, \\
& \tilde{f}_{m}\left(\frac{1}{\sqrt{2}}\right)=f\left(\frac{1}{\sqrt{2}}\right)-\frac{2 m-1}{1 / \sqrt{2}} K=\frac{\sqrt{2}}{2} K-(2 m-1) \sqrt{2} K \\
& =\sqrt{2} K\left(\frac{1}{2}-2 m+1\right)=\sqrt{2} K\left(\frac{3}{2}-2 m\right)<0, \\
& \tilde{f}_{m}^{\prime}\left(\frac{1}{\sqrt{2}}\right)=\underbrace{f^{\prime}\left(\frac{1}{\sqrt{2}}\right)}_{+\infty}+\lim _{\gamma \rightarrow 1 / \sqrt{2}} \frac{(2 m-1) K}{\gamma^{2}}=\infty \text {. }
\end{aligned}
$$

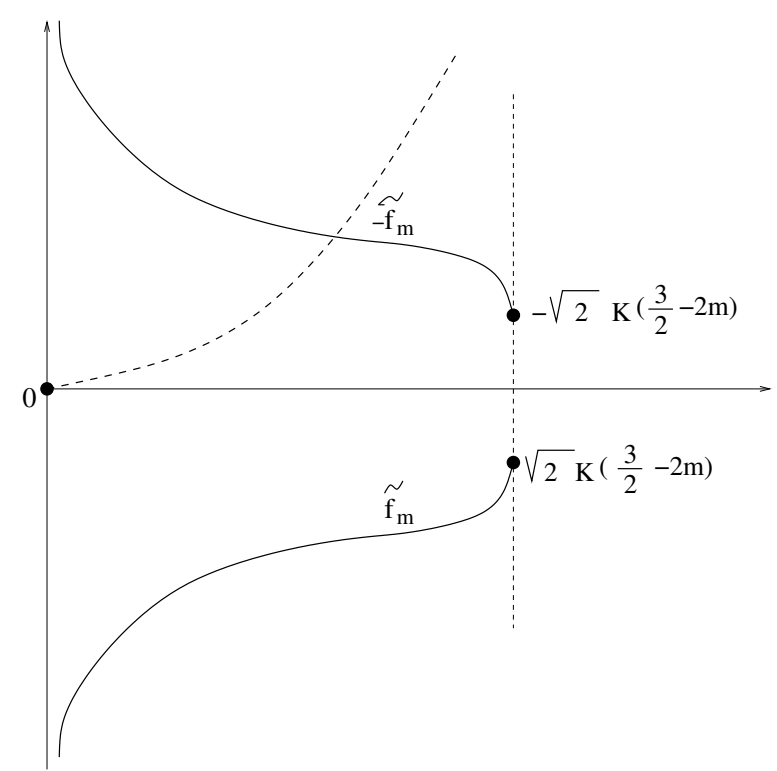

Figure 4: The graphs of $\tilde{f}_{m}$ and $-\tilde{f}_{m}$.

The graphs of $\tilde{f}_{m}$ and $-\tilde{f}_{m}$ are given in Figure 4 . One may observe that

$$
f_{m}(1 / \sqrt{2})=-\tilde{f}_{m+1}(1 / \sqrt{2}) .
$$


Indeed,

$$
\begin{aligned}
\frac{1}{2}+2 m & =-\frac{3}{2}+2 m+2 \\
\Leftrightarrow\left(\frac{1}{2}+2 m\right) \sqrt{2} K & =-\left(\frac{3}{2}-2(m+1)\right) \sqrt{2} K \\
\Leftrightarrow f_{m}(1 / \sqrt{2}) & =-\tilde{f}_{m+1}(1 / \sqrt{2}) .
\end{aligned}
$$

Hence the graphs of $f_{m}$ and $-\tilde{f}_{m+1}$ match at $1 / \sqrt{2}$. See Figure 5 . The first few values for $m=0,1,2$ are

$$
\begin{aligned}
f_{0}(1 / \sqrt{2}) & =f(1 / \sqrt{2})=\frac{K}{\sqrt{2}}, \\
f_{1}(1 / \sqrt{2}) & =\frac{9 K}{\sqrt{2}} \\
f_{2}\left(\frac{1}{\sqrt{2}}\right) & =\frac{13 K}{\sqrt{2}} .
\end{aligned}
$$

Hence for any $\mathbf{y} \geq 0$ at least one of the equations

$$
\begin{gathered}
\frac{12 \mathbf{y}}{\mathbf{x}^{3}} \gamma^{2}=f_{m}(\gamma), \\
\frac{12 \mathbf{y}}{\mathbf{x}^{3}} \gamma^{2}=-\tilde{f}_{m}(\gamma)
\end{gathered}
$$

has a solution.

If $\frac{6 \mathbf{y}}{\mathbf{x}^{3}}$ is small enough (for instance smaller than $K / \sqrt{2}$ ), then there is an unique solution. The complete picture is given by Figure 5 . 


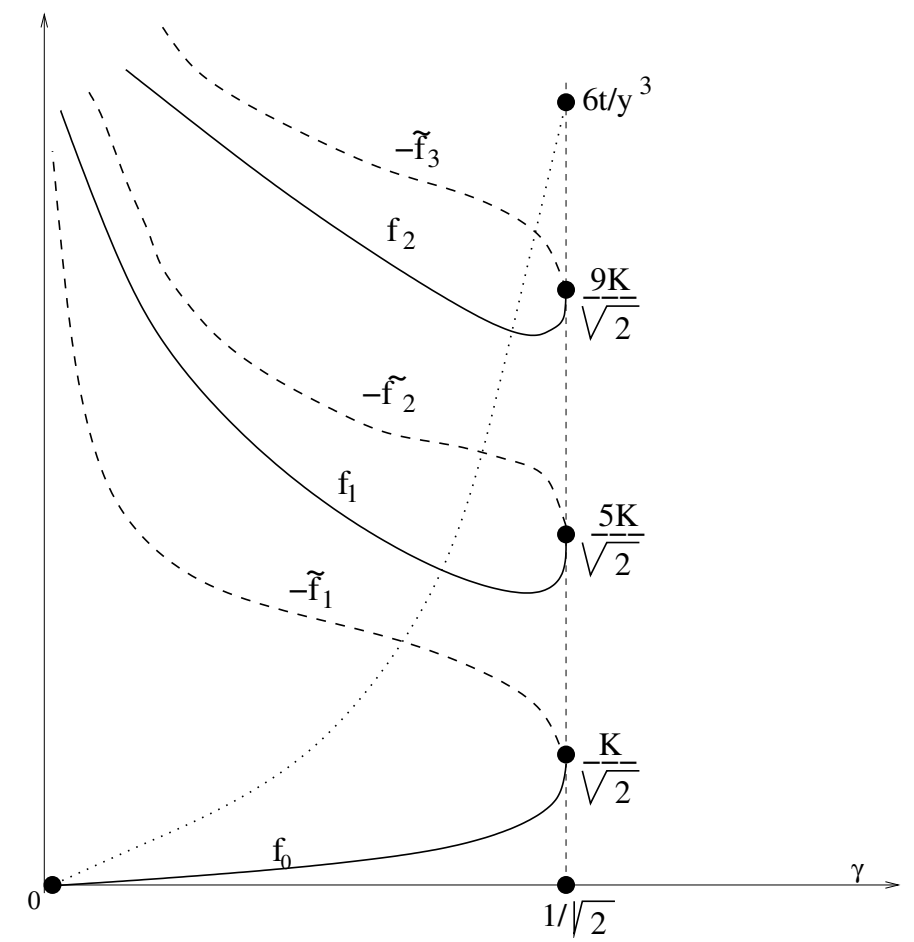

Figure 5: The graphs of $\left(f_{0},-\tilde{f}_{1}\right),\left(f_{1},-\tilde{f}_{2}\right),\left(f_{2},-\tilde{f}_{3}\right)$ match at $1 / \sqrt{2}$.

As

$f_{1}(1 / \sqrt{2})-f_{0}(1 / \sqrt{2})=f_{2}(1 / \sqrt{2})-f_{1}(1 / \sqrt{2})=\cdots=f_{n+1}(1 / \sqrt{2})-f_{n}(1 / \sqrt{2})=\frac{4 K}{\sqrt{2}}$,

the number of intersections will be

$$
2\left[\frac{\frac{6 \mathbf{y}}{\mathbf{x}^{3}}-\frac{K}{\sqrt{2}}}{\frac{4 K}{\sqrt{2}}}\right]=2\left[\frac{3}{\sqrt{2} K} \cdot \frac{\mathbf{y}}{\mathbf{x}^{3}}-\frac{1}{4}\right],
$$

where $[x]$ denotes the greatest integer smaller than $x$.

We arrived at the following result.

Theorem 5.1 Let $\mathbf{y} / \mathbf{x}^{3}>0$. There are finitely many geodesics between the origin and $(\mathbf{x}, \mathbf{y})$ with $\mathbf{x} \neq 0$.

If $\mathbf{y} / \mathbf{x}^{3}$ is small enough there is only one geodesic. 
If $\mathbf{y} / \mathbf{x}^{3}$ is large enough, the number $N$ of geodesics is approximated by

$$
N \approx 2\left[\frac{3}{\sqrt{2} K} \cdot \frac{\mathbf{y}}{\mathbf{x}^{3}}-\frac{1}{4}\right] .
$$

\section{References}

1. A. Agrachev, B. Bonnard, M. Chyba and I. Kupka: Sub-Riemannian Sphere in Martinet Flat Case, Control, Optimization and Calculus of Variations, 2 (1997), 377-448.

2. R. Beals, B. Gaveau and P.C. Greiner: Subelliptic Geometry, in: E. Ramirez de Arellano et al.(Eds.), Operator Theory for Complex and Hypercomplex Analysis, Contemporary Mathematics, 212, Amer. Math. Soc., Providence, 1998, 25-39.

3. R. Beals, B. Gaveau and P.C. Greiner: Complex Hamiltonian Mechanics and Parametrices for Subelliptic Laplacians,I, II, III, Bull. Sci. Math., 121 (1997), 1-36, 97-149, 195-259.

4. R. Beals, B. Gaveau and P.C. Greiner: On a Geometric Formula for the Fundamental Solution of Subelliptic Laplacians, Math. Nachr., 181 (1996), 81-163.

5. R. Beals, B. Gaveau and P.C. Greiner: The Green Function of Model Step Two Hypoelliptic Operators and the Analysis of Certain Tangential Cauchy Riemann Complexes, Advances in Math., 121 (1996), 288-345.

6. R. Beals, B. Gaveau and P.C. Greiner: Hamilton-Jacobi Theory and the Heat Kernel on Heisenberg Groups, J.Math. Pures Appl., 79 \#7, (2000) 633-689

7. B. Bonnard and M. Chyba: Sub-Riemannian Geometry: The Martinet Case, Canadian Math. Soc. Conf. Proc., 25, 1998.

8. O. Calin, D.C. Chang and P.C. Greiner: On a Step $2(k+1)$ subRiemannian manifold, to appear in the J. Geometric Analysis, (2004).

9. O. Calin, D.C. Chang, P.C. Greiner and Y. Kannai: On the geometry induced by a Grushin operator, preprint, (2004). 
10. O. Calin and D.C. Chang : Heat kernel for highly degenerate Grushin operators, preprint, (2004).

11. W.L. Chow: Uber Systeme van Linearen Partiellen Differentialgleichungen erster Ordnung, Math. Ann., 117 (1939), 98-105

12. B. Gaveau: Principe de moindre action, propagation de la chaleur et estimées sous-elliptiques sur certains groupes nilpotent, Acta Math., 139 (1977), 95-153.

13. V.V. Grushin: On a class of hypoelliptic operators, Math. Sbornik. 83 \#125 (1970), 456-473.

14. L. Hörmander: Hypoelliptic Second Order Differential Equations, Acta Math., 119 (1975), 141-171.

15. D.F. Lawden: Elliptic functions and Applications, Applied Math. Sciences 80, Springer-Verlag, 1989

16. R. Strichartz: Subriemannian Geometry, J. Diff. Geometry, 24 (1986), 221-263. 